\title{
Strategic Argumentation under Grounded Semantics is NP-Complete
}

\author{
Guido Governatori ${ }^{1}$, Michael J. Maher ${ }^{2}$, Francesco Olivieri ${ }^{3}$, \\ Antonino Rotolo ${ }^{4}$ and Simone Scannnapieco ${ }^{3}$ \\ 1 NICTA Queensland, Australia ${ }^{\star}$ \\ 2 UNSW, Canberra \\ 3 Univeristy of Verona \\ 4 CIRSFID and Law Faculty, University of Bologna
}

\begin{abstract}
We study the complexity of the Strategic Argumentation Problem for 2-player dialogue games where a player should decide what move to play at each turn in order to prove (disprove) a given claim. We shall prove that this is an NP-complete problem. The result covers one the most popular argumentation semantics proposed by Dung [4]: the grounded semantics.
\end{abstract}

\section{Introduction}

Consider the following argument exchange due to [13], where two players are involved, a proponent $\mathrm{Pr}$ and an opponent $\mathrm{Op}$ :

$\operatorname{Pr}_{0}$ : "You killed the victim."

$\mathrm{Op}_{1}$ : "I did not commit murder! There is no evidence!"

$\operatorname{Pr}_{1}$ : "There is evidence. We found your ID card near the scene."

$\mathrm{Op}_{2}$ : "It's not evidence! I had my ID card stolen!"

$\mathrm{Pr}_{2}$ : "It is you who killed the victim. Only you were near the scene at the time of the murder."

$\mathrm{Op}_{3}$ : "I didn't go there. I was at facility A at that time."

$\mathrm{Pr}_{3}$ : "At facility A? Then, it's impossible to have had your ID card stolen since facility A does not allow a person to enter without an ID card."

The peculiarity of this argument game is that the exchange of arguments reflects an asymmetry of information between the two parties. First, each player does not know the other player's knowledge, thus she cannot predict neither which arguments will be attacked, nor which counterarguments may be employed for attacking the arguments. Second, the private information disclosed by a party might be eventually used by the adversary to construct and play justified counterarguments. In the previous setting, $\mathrm{Pr}_{3}$ attacks $\mathrm{Op}_{2}$, but only after $\mathrm{Op}_{3}$ has been given. That is to say, the attack $\mathrm{Pr}_{3}$ of the proponent is possible only when the opponent discloses some private information through the move $\mathrm{Op}_{3}$.

* NICTA is funded by the Australian Government through the Department of Communications and the Australian Research Council through the ICT Centre of Excellence Program. 
The scenario above exemplifies argument games with incomplete information, i.e., dialogues where the structure of the game is not common knowledge among the players. Dialogues with incomplete information are typical in domains such as the legal domain where a disputant does not know what arguments her opponent will employ. As argued in [7], players have different logical theories which constitute their private knowledge, being unknown by the opposite party. A player may build an argument supporting her claim by using some of her private knowledge; in turn, the other party may then reuse such rules and others (again from her own private knowledge) to construct a new argument defeating the previously constructed argument. In other words, the set $R$ of rules that are used to build arguments is partitioned into three subsets: a set $R_{\text {Com }}$ known by both players and two subsets $R_{\mathrm{Pr}}$ and $R_{\mathrm{Op}}$ corresponding, respectively, to Pr's and Op's private knowledge. Consider a setting where $F=\{a, d, f\}$ is the known set of facts, $R_{\text {Com }}=\emptyset$, and the players have the following rules:

$$
\begin{aligned}
R_{\mathrm{Pr}} & =\{a \Rightarrow b, \quad d \Rightarrow c, \quad c \Rightarrow b\} \\
R_{\mathrm{Op}} & =\{c \Rightarrow e, \quad e, f \Rightarrow \neg b\} .
\end{aligned}
$$

If Pr's intent is to prove $b$ and she plays $\{a \Rightarrow b\}$, then $\operatorname{Pr}$ wins the game. If $\operatorname{Pr}$ plays $\{d \Rightarrow c, c \Rightarrow b\}$ (or even $R_{\operatorname{Pr}}$ ), this allows Op to succeed. Here, a minimal subset of $R_{\operatorname{Pr}}$ is successful. The situation can be reversed for Pr. Replace the sets of private rules with

$$
\begin{aligned}
R_{\mathrm{Pr}} & =\{a \Rightarrow b, \quad d \Rightarrow \neg c\} \\
R_{\mathrm{Op}} & =\{d, c \Rightarrow \neg b, \quad f \Rightarrow c\} .
\end{aligned}
$$

In this second case, the move $\{a \Rightarrow b\}$ is not successful for $\operatorname{Pr}$, while playing with the whole $R_{\operatorname{Pr}}$ ensures victory.

[7] has considered standard propositional Defeasible Logic [2] in a dialogue game to represent the knowledge of the players, the structure of the arguments, and to perform reasoning. In this setting, it has been proved that the problem of deciding what set of rules to successfully play (called Strategic Argumentation Problem) at a given turn is NP-complete. That result was offering an interesting starting point, but it covers a non-standard argumentation semantics [6]. In this paper, we shall extend that result by considering one of the most popular argumentation semantics proposed by Dung [4], i.e., grounded semantics.

The layout of the paper is as follows. Section 2 recalls the basics of [4]'s grounded semantics and a framework for argumentation with logically structured arguments for that semantics. Section 3 considers the strategic argumentation framework introduced in [7] and offers a formulation of the Strategic Argumentation Problem. Section 4 outlines Defeasible Logic and, in particular, the Ambiguity Propagating Defeasible Logic variant that corresponds to Dung's grounded semantics. Section 5 presents an implementation of the strategic argumentation game with Defeasible Logic as the underlying logical framework, and Section 6 proves the main complexity results. Some conclusions end the paper. 


\section{Abstract Argumentation and Grounded Semantics}

The well-known, abstract argumentation paradigm was originally proposed by Dung [4] to study the general aspects of argumentation without specifying the internal structure of arguments. In this perspective, an argumentation framework $A F$ is a structure $\langle\mathscr{A}, \gg\rangle$, where $\mathscr{A}$ is a non-empty set of arguments and $\gg$ is a binary attack relation on $\mathscr{A}$. For any pair or arguments $A$ and $B$ in $\mathscr{A}, A \gg B$ means that $A$ attacks $B$. If the goal is to determine whether an argument can be accepted, this cannot be done by only choosing between two arguments that directly conflict with each other. We need to understand how arguments can be indirectly defended by other arguments. The corresponding literature flourishes [3] with different formalisations among which Dung's grounded semantics is perhaps the most popular one.

Let us recall the basic formal concepts of abstract argumentation and the basic features of Dung's grounded semantics. An argument $A$ is acceptable w.r.t. a set of arguments $\mathscr{S}$ if and only if any argument defeating $A$ is defeated by an argument in $\mathscr{S}$. The function $F_{A F}$, for an argumentation framework $A F=\langle\mathscr{A}, \gg\rangle$, is defined as $F_{A F}: 2^{\mathscr{A}} \Rightarrow 2^{\mathscr{A}}$ and $F_{A F}(\mathscr{S})=\{A \mid A$ is acceptable w.r.t. $\mathscr{S} \subseteq \mathscr{A}\}$. A grounded extension $G E(A F)$ of an argumentation framework $A F$ is the least fixed-point of $F_{A F}$. An $\operatorname{argument} A$ and its conclusion are justified w.r.t. an argumentation framework $A F$ if, and only if, $A \in G E(A F)$.

As is done in $[6,12,14]$, given the above framework the (internal) logical structure of arguments can be specified in such a way that arguments are logical inference trees.

Definition 1. The language consists of literals and rules. Given a set PROP of propositional atoms, the set of literals is Lit $=\mathrm{PROP} \cup\{\neg p \mid p \in \mathrm{PROP}\}$. We denote with $\sim p$ the complementary of literal $p$; if $p$ is a positive literal $q$, then $\sim p$ is $\neg q$, and if $p$ is $a$ negative literal $\neg q$, then $\sim p$ is $q$.

Let $\mathrm{Lab}$ be a set of unique labels. A rule $r$ with $r \in \mathrm{Lab}$ describes the relation between a subset of Lit, called the antecedent or the premises of $r$ and denoted by $A(r)$ (which may be empty) and a literal in Lit, called the consequent or head of $r$ and denoted by $C(r)$. Three kind of rules are allowed: strict rules of the form $r: A(r) \rightarrow C(r)$, defeasible rules of the form $r: A(r) \Rightarrow C(r)$, and defeaters of the form $r: A(r) \rightsquigarrow C(r)$. An undisputed fact is represented as a strict rule with empty antecedent.

A strict rule is a rule in the classical sense: whenever the antecedent holds, so is the conclusion. A defeasible rule is allowed to assert its conclusion unless there is contrary evidence to it. A defeater is a rule that cannot be used to draw any conclusion, but can provide contrary evidence to complementary conclusions.

Definition 2. An argumentation theory $D$ is a structure $(R,>)$, where $R$ is a (finite) set of rules and $>\subseteq R \times R$ is a binary relation on $R$ called the superiority relation.

The relation $>$ describes the relative strength of rules, that is to say, when a single rule may override the conclusion of another rule, and is required to be irreflexive, asymmetric and acyclic (i.e., its transitive closure is irreflexive).

By combining the rules in a theory, we can build arguments (we adjust the definition in [12] to meet Definition 2). In what follows, for a given argument $A$, Conc returns its 
conclusion, Sub returns all its sub-arguments, Rules returns all the rules in the argument and, finally, TopRule returns the last inference rule in the argument.

Definition 3 (Argument). Let $D=(R,>)$ be an argumentation theory and $\Rightarrow \in\{\rightarrow, \Rightarrow$ $, \rightsquigarrow\}$. An argument $A$ constructed from $D$ has the form $A_{1}, \ldots, A_{n} \Rightarrow_{r} \phi$, where

- $A_{k}$ is an argument constructed from $D$, for $1 \leq k \leq n$, and

- $r$ : Conc $\left(A_{1}\right), \ldots, \operatorname{Conc}\left(A_{n}\right) \Rightarrow \phi$ is a rule in $R$.

With regard to argument $A$, the following holds:

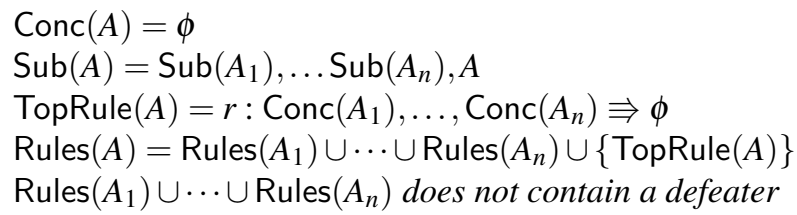

The following example illustrates the notions just introduced.

Example 1. Given the set

$$
R=\left\{r_{1}: \rightarrow a, \quad r_{2}: \Rightarrow b, \quad r_{3}: a, b \Rightarrow c\right\}
$$

we have arguments:

$$
\begin{array}{ll}
A_{1}: \quad \rightarrow_{r_{1}} a & \text { (strict argument) } \\
A_{2}: \quad \Rightarrow_{r_{2}} b & \text { (defeasible argument) } \\
A_{3}: A_{1}, A_{2} \Rightarrow_{r_{3}} c & \text { (defeasible argument) }
\end{array}
$$

Let us now consider conflicts between arguments. In grounded semantics we just need to consider rebuttals. Hence, conflicts between contradictory argument conclusions are resolved on the basis of preferences over arguments using a simple last-link ordering according to which an argument $A$ is stronger than another argument $B$, denoted as $A>B$, if, and only if, the rule $\operatorname{TopRule}(A)$ is stronger than the rule $\operatorname{Top} \operatorname{Rule}(B)$ (i.e. TopRule $(A)>$ TopRule $(B))$.

Definition 4 (Defeats). An argument $B$ defeats an argument $A$ if, and only if $\exists A^{\prime} \in$ $\operatorname{Sub}(A)$ such that $\operatorname{Conc}(B)=\sim \operatorname{Conc}\left(A^{\prime}\right)$, and $A^{\prime} \ngtr B$.

We can now define the argumentation framework that is determined by an argumentation theory.

Definition 5 (Argumentation Framework). Let $D=(R,>)$ be an argumentation theory. The argumentation framework determined by $D$ is $\langle\mathscr{A}, \gg\rangle$ where $\mathscr{A}$ is the set of all arguments constructed from $D$, and $\gg$ is the defeat relation defined above.

Given this definition of argumentation framework, if $D$ is an argumentation theory, we can abuse notation somewhat and write $G E(D)$ to denote the grounded extension of the argumentation framework determined by $D$.

The following is thus a standard result that can be obtained:

Theorem 1. Given a theory $D$, a conclusion $\phi$ is justified by $D$ under the grounded semantics iff there is an argument $A$ in $G E(D)$ such that $\operatorname{Conc}(A)=\phi$. 


\section{Strategic Argumentation}

We herein consider the strategic argumentation framework introduced in [7]. The dialogue games proposed in that work involves an alternating sequence of interactions between two players, the Proponent $\mathrm{Pr}$ and the Opponent Op. The content of the dispute being that $\operatorname{Pr}$ attempts to assess the validity of a particular claim, whereas Op attacks Pr's claims in order to refute such a claim. In that setting, Op has the burden of proof on the opposite claim, and not just the duty to refute Pr's claim.

The challenge between the parties is formalised by means of argument exchange. In the majority of concrete instances of argumentation frameworks, arguments are defined as chains of reasoning based on facts and rules captured in some formal language. Each party adheres to a particular set of game rules as defined below.

The players partially share knowledge of a logical theory, which includes common facts and rules to put forward arguments. Each participant has a private knowledge regarding some rules of the theory. Other rules are known by both parties, but the set of such rules may be empty. These rules along with all the facts of the theory represent the common knowledge of both participants.

The repertoire of moves at each turn just includes either putting forward an argument, or passing. By putting forward a private argument during a step of the game, the agent increases the common knowledge by the rules used within the argument just played. Essentially, the agent chooses a subset of her private knowledge which, along with the current common knowledge, justifies her claim. On the other hand, when a player passes, she declares her defeat and the game ends. This happens when there is no combination of the remaining private rules which proves her claim.

We now provide the formal definition of a dialogue game. The state of the game at turn $i$ is denoted by a theory $D^{i}=\left(R_{\text {com }}^{i},>\right)$ and by two sets $R_{\mathrm{Pr}}^{i}$ and $R_{\mathrm{Op}}^{i} \cdot R_{\mathrm{Com}}^{i}$ is the set of rules known by both participants at turn $i$ (which may be empty when $i=0$ ), and $R_{\mathrm{Pr}}^{i}\left(R_{\mathrm{Op}}^{i}\right)$ is the private knowledge of $\operatorname{Pr}(\mathrm{Op})$ at turn $i$. We assume that each player is informed about the restriction of $>$ to the rules that she knows. We assume that the private theories of the proponent and the opponent are conflict-free.

We now formalise the game rules which establish how the theory $D^{i-1}$ and the sets $R_{\mathrm{Pr}}^{i-1}, R_{\mathrm{Op}}^{i-1}$ are modified based on the move played at turn $i$.

The parties start the game by choosing the content of dispute $l$ (in our case, a literal) to discuss about. At turn $i, \operatorname{Pr}$ has the burden to justify $l$ (under the grounded semantics) by using the current common knowledge along with a subset of $R_{\mathrm{Pr}}^{i-1}$, whereas Op's final goal is to justify $\sim l$ (under the grounded semantics) using $R_{\mathrm{Op}}^{i-1}$ instead of $R_{\mathrm{Pr}}^{i-1}$. We point out that at turn $i, \operatorname{Pr}(\mathrm{Op})$ may put forward an argument whose terminal literal differs from $l(\sim l)$.

Let $R_{\mathrm{Com}}^{0}, R_{\mathrm{Pr}}^{0}$ and $R_{\mathrm{Op}}^{0}$ be respectively the common knowledge, and the private knowledge of $\operatorname{Pr}$ and Op at the beginning of the game, and $D^{0}=\left(R_{c o m}^{0},>\right)$. If $l$ is justified by $D^{0}$ then Op starts the game. Otherwise, Pr does so.

At turn $i$, if $\operatorname{Pr}$ plays $R^{i}$, then

- $\sim l$ is justified by $D^{i-1}$ under the grounded semantics;

$-R^{i} \subseteq R_{\mathrm{Pr}}^{i-1}$

- $D^{i}=\left(R_{\text {Com }}^{i},>\right)$; 
- $R_{\mathrm{Pr}}^{i}=R_{\mathrm{Pr}}^{i-1} \backslash R^{i}, R_{\mathrm{Op}}^{i}=R_{\mathrm{Op}}^{i-1}$, and $R_{\mathrm{Com}}^{i}=R_{\mathrm{Com}}^{i-1} \cup R^{i}$;

- $l$ is justified by $D^{i}$ under the grounded semantics.

At turn $i$, if Op plays $R^{i}$, then

$-l$ is justified $D^{i-1}$ under the grounded semantics;

- $R^{i} \subseteq R_{\text {Op }}^{i-1}$

- $D^{i}=\left(R_{\text {Com }}^{i},>\right)$;

- $R_{\mathrm{Pr}}^{i}=R_{\mathrm{Pr}}^{i-1}, R_{\mathrm{Op}}^{i}=R_{\mathrm{Op}}^{i-1} \backslash R^{i}$, and $R_{\mathrm{Com}}^{i}=R_{\mathrm{Com}}^{i-1} \cup R^{i}$;

- $\sim l$ is justified by $D^{i}$ under the grounded semantics.

The corresponding decision problem can be formulated as follows:

\section{Strategic argumentation problem under grounded semantics}

Pr'S INSTANCE FOR TURN $i$ : Let $l$ be the content of dispute, $R_{\mathrm{Pr}}^{i-1}$ be the set of the private rules of $\operatorname{Pr}$, and $D^{i-1}$ be such that $\sim l$ is justified by $D^{i-1}$ under the grounded semantics.

QUESTION: Is there a subset $R^{i}$ of $R_{\mathrm{Pr}}^{i-1}$ such that $l$ is justified by $D^{i}$ under the grounded semantics?

Op's instance of the problem is similar, asking for $\sim l$ to be justified.

Later, we will show that both Pr's and Op's version of this problem is NP-complete.

\section{Defeasible Logic}

Defeasible Logic is a rule-based skeptical approach to nonmonotonic reasoning. It is based on a logic programming-like language and is simple, efficient but flexible formalism capable of dealing with many intuitions of non-monotonic reasoning in a natural and meaningful way [1].

The language of Defeasible Logic consists of literals and rules. In order to avoid notational redundancies, from now on we use the same definitions of PROP, Lit, complementary literal, and the same rule types, structure and notation as already introduced in Definition 1.

A defeasible theory $D$ is a triple $(F, R,>)$, where $F \subseteq$ Lit is a set of indisputable statements called facts, $R$ is a (finite) set of rules, and $>\subseteq R \times R$ is a superiority relation on $R$ as introduced in Definition 2.

The proof theory of a defeasible logic can draw two main types of conclusions. A conclusion $+d f q$ expresses that the literal $q$ can be proven defeasibly, while a conclusion $-d f q$ expresses that a proof has established that $q$ cannot be proven defeasibly. $d f$ is known as a tag. Different tags are used to characterize the conclusions of different defeasible logics. In this paper, there are two defeasible logics of interest, characterized by the tags $\partial$ and $\delta$.

The logics are denoted by $\operatorname{DL}(\partial)$ and $\operatorname{DL}(\delta)$. There is no room here to provide the full inference rules for these logics; we refer the reader to [5] (where $\partial_{a p}$ is used instead of $\delta$ ). 
In a series of papers $[8,9]$ Maher investigates the relative expressiveness of variants of Defeasible Logic. Briefly, two (defeasible) logics $L_{1}$ and $L_{2}$ have the same expressiveness iff the two logics can simulate each other. A defeasible logic $L_{2}$ simulates a defeasible $\operatorname{logic} L_{1}$ if there is a polynomial time transformation $T$ that transforms a theory $D_{1}$ of $L_{1}$ to a theory $D_{2}=T\left(D_{1}\right)$ of $L_{2}$ such that, for any set of additional facts $F, D_{1}+F$ and $D_{2}+F$ have the same conclusions in the language of $D_{1}{ }^{1}$. [9] provides polynomial time transformations from $\operatorname{DL}(\partial)$ to $\operatorname{DL}(\delta)$ and the other way around.

Theorem 2. [9] $\mathrm{DL}(\delta)$ can simulate $\mathrm{DL}(\partial)$, and vice versa.

\section{Strategic Argumentation in DL}

A defeasible logic implementation of the game introduced in Section 3 follows the same structure, but some specifics are expressed in terms of the defeasible logic. From now on, the content of the dispute discussed by the players will be called the critical literal, and the arguments brought about by the players will be in the form of defeasible derivations. We state that the players may not present arguments in parallel, that is to say, they take turns in making their move.

The state of the game at turn $i$ is now denoted by a defeasible theory $D^{i}=\left(F, R_{c o m}^{i},>\right)$ and by two sets $R_{\mathrm{Pr}}^{i}$ and $R_{\mathrm{Op}}^{i} \cdot R_{\mathrm{Com}}^{i}$ is the set of rules known by both participants at turn $i$ (which may be empty when $i=0$ ), and $R_{\mathrm{Pr}}^{i}\left(R_{\mathrm{Op}}^{i}\right)$ is the private knowledge of $\operatorname{Pr}(\mathrm{Op})$ at turn $i$. We assume that each player is informed about the restriction of $>$ to the rules that she knows. $D^{i}$ is assumed to be coherent and consistent for each $i$, i.e., there is no literal $p$ such that: (i) $p$ is at the same time defeasibly proved and refuted in $D^{i}$, and (ii) both $p$ and $\sim p$ are defeasibly proved in $D^{i}$.

The game rules discussed in Section 3 are instantiated as follows. In place of requiring that a literal is justified under the grounded semantics, we instead require that it can be inferred defeasibly from the defeasible theory under the given logic.

Thus we formulate the strategic argumentation problem under a defeasible logic as follows.

\section{Strategic argumentation problem under $\mathbf{D L}(d f)$}

Pr's INSTANCE FOR TURN $i$ : Let $l$ be the critical literal, $R_{\operatorname{Pr}}^{i-1}$ be the set of the private rules of $\operatorname{Pr}$, and $D^{i-1}$ be such that either $D^{i-1} \vdash-d f l$ if $i=1$, or $D^{i-1} \vdash+d f \sim l$ otherwise.

QUESTION: Is there a subset $R^{i}$ of $R_{\mathrm{Pr}}^{i-1}$ such that $D^{i} \vdash+d f l$ ?

\section{NP-Completeness Result}

We are now ready to give the result of the paper, namely deciding what argument to play in a given turn of a dialogue game under Dung's grounded semantics is an NP-complete problem even when the problem of deciding whether a conclusion follows from an argument is computable in polynomial time.

Governatori et al. [7] proved that the same problem is NP-complete for DL with ambiguity blocking, i.e., $\operatorname{DL}(\partial)$.

\footnotetext{
${ }^{1} D+F$ denotes the addition of the facts $F$ to the facts in the defeasible theory $D$.
} 
Theorem 3. [7] The strategic argumentation problem under $\mathrm{DL}(\partial)$ is NP-complete.

While it is possible to define $\operatorname{DL}(\partial)$ in terms of an argumentation semantics, the logic corresponding to Dung's grounded semantics is $\operatorname{DL}(\delta)[6]$. Thus the next step is to determine the computational complexity of the problem at hand for $\operatorname{DL}(\delta)$.

We cannot employ Theorem 2 directly, because it only applies to addition of facts. However, it is shown in [10] that every strategic argumentation problem involving addition of rules can be easily transformed into an equivalent strategic argumentation problem only involving addition of facts. Thus we can apply Theorem 2 to show that the transformed strategic argumentation problem for $\operatorname{DL}(\partial)$ can be reduced to the corresponding transformed problem for $\mathrm{DL}(\delta)$. The NP-completeness of the strategic argumentation problem under $\operatorname{DL}(\delta)$ now follows.

Theorem 4. The strategic argumentation problem under $\mathrm{DL}(\boldsymbol{\delta})$ is NP-complete.

In [6] the equivalence of derivations in $\operatorname{DL}(\delta)$ and justified conclusions under grounded semantics has been established.

Theorem 5. [6] Given a defeasible theory $D, D \vdash+\delta l$ iff $l$ is justified by $D$ under the grounded semantics.

We can solve the strategic argumentation problem by non-deterministically choosing a set $R^{i}$ of rules and then verifying whether the critical literal $l$ is justified in the argumentation framework determined by $D^{i}$, or not. Further, the literals justified by the grounded semantics are computable in polynomial time. Thus the strategic argumentation problem is in NP.

Now, from Theorem 4 and Theorem 5 we obtain the main result of this contribution.

Theorem 6. The strategic argumentation problem under the grounded semantics is NP-complete.

\section{Conclusions}

Almost all research in $\mathrm{AI}$ on argumentation assumes that strategic dialogues are games of complete information, that is where the structure of the game is common knowledge among the players (see [7] for a review of the literature). Following [11,13], we argued that argument games work under incomplete information: by not knowing the other player's knowledge, each player cannot predict neither which arguments will be attacked, nor which counterarguments will be employed for attacking her arguments. We proved that the problem of deciding what set of rules to play at a given move is NP-complete, even if the problem of deciding whether a given theory (defeasibly) entails a literal can be computed in polynomial time.

\section{References}

1. Antoniou, G.: A Discussion of Some Intuitions of Defeasible Reasoning. In: Vouros, G., Panayiotopoulos, T. (eds.) Methods and Applications of Artificial Intelligence, Lecture Notes in Computer Science, vol. 3025, pp. 311-320. Springer Berlin / Heidelberg (2004) 
2. Antoniou, G., Billington, D., Governatori, G., Maher, M.J.: Representation results for defeasible logic. ACM Transactions on Computational Logic 2(2), 255-286 (2001)

3. Baroni, P., Giacomin, M.: Semantics of abstract argument systems. In: Argumentation in Artificial Intelligence, pp. 25-44. Springer Berlin/Heidelberg (2009)

4. Dung, P.M.: On the acceptability of arguments and its fundamental role in nonmonotonic reasoning, logic programming and n-person games. Artif. Intell. 77(2), 321-358 (1995)

5. Governatori, G., Maher, M.J., Antoniou, G., Billington, D.: Argumentation semantics for defeasible logic. J. Log. Comput. 14(5), 675-702 (2004)

6. Governatori, G., Maher, M.J., Antoniou, G., Billington, D.: Argumentation Semantics for Defeasible Logics. Journal of Logic and Computation 14(5), 675-702 (2004)

7. Governatori, G., Olivieri, F., Scannapieco, S., Rotolo, A., Cristani, M.: Strategic argumentation is NP-complete. In: Proc. ECAI 2014. IOS Press (2014)

8. Maher, M.J.: Relative expressiveness of defeasible logics. Theory and Practice of Logic Programming 12(4-5), 793-810 (2012)

9. Maher, M.J.: Relative expressiveness of defeasible logics II. Theory and Practice of Logic Programming 13, 579-592 (2013)

10. Maher, M.J.: Complexity of exploiting privacy violations in strategic argumentation. In: Proc. Pacific Rim International Conf. on Artificial Intelligence. Lecture Notes in Computer Science, vol. 8862, pp. 523-535. Springer (2014)

11. Okuno, K., Takahashi, K.: Argumentation system with changes of an agent's knowledge base. In: IJCAI'09. pp. 226-232 (2009)

12. Prakken, H.: An abstract framework for argumentation with structured arguments. Argument and Computation 1(2), 93-124 (2010)

13. Satoh, K., Takahashi, K.: A semantics of argumentation under incomplete information. In: Proceedings of Jurisn 2011 (2011)

14. Toni, F.: A generalised framework for dispute derivations in assumption-based argumentation. Artificial Intelligence 195, 1-43 (2013) 